\title{
Transmission Electron Microscopy Specimen Preparation of Delicate Materials Using Tripod Polisher
}

\author{
Hyun-Woo Cha, Min-Chul Kang, Keesam Shin ${ }^{1}$, Cheol-Woong Yang* \\ School of Advanced Materials Science \& Engineering, Sungkyunkwan University, Suwon 16419, Korea \\ ${ }^{1}$ School of Nano and Advanced Materials Engineering, Changwon National University, Changwon 51140, Korea
}

*Correspondence to:
Yang CW,
Tel: +82-31-290-7362
Fax: +82-31-290-7371
E-mail: cwyang@skku.edu

Received May 30, 2016

Revised June 23, 2016

Accepted June 23, 2016

\begin{abstract}
Transmission electron microscopy (TEM) is a powerful tool for analyzing a broad range of materials and provides localized information about the microstructure. However, the analysis results are strongly influenced by the quality of the thin foil specimen. Sample preparation for TEM analysis requires considerable skill, especially when the area of interest is small or the material of interest is difficult to thin because of its high hardness and its mechanical instability when thinned. This article selectively reviews recent advances in TEM sample preparation techniques using a tripod polisher. In particular, it introduces two typical types (flat type and wedge type) of TEM sample preparation and the benefits and drawbacks of each method; finally, a method of making better samples for TEM analysis is suggested.
\end{abstract}

Key Words: Tripod polisher, Flat-type polishing, Wedge-type polishing, Hard materials, Sample preparation

\section{INTRODUCTION}

Transmission electron microscopy (TEM), which offers a wide variety of imaging and analytical techniques, plays a significant role in the investigation of materials and is a very powerful characterization instrument (Srot et al., 2014). Because of these useful functions of TEM, TEM analysis has been extensively used in various fields of materials science and engineering. In particular, the semiconductor industry uses TEM not only as a research tool but also for quality control (Chen \& Ivey, 2002). Successful TEM analysis depends on many parameters, including the preparation of thin foil specimens. Despite amazing advances in equipment performance, hands-on experience is still important for preparing successful TEM specimens, and the quality of a thin foil specimen strongly affects the final analysis result. Many studies describe how to prepare TEM specimens (Ayache et al., 2010; Barna et al., 1997; Goodhew, 1985;
Hirsch et al., 1965; Williams \& Carter, 2009). Each material and each field of electron microscopy may require its own specific preparation technique. It is not possible to cover all methods in this article. Instead, this article focuses on mechanical polishing using a tripod polisher. The tripod polishing method was originally developed to prepare sitespecific TEM specimens of Si semiconductor devices with a large transparent area (Klepeis et al., 1987). Application of the method has been extended to a broader range of hard materials such as other types of semiconductors and ceramics or composite materials (Ayache et al., 2010). For these types of materials, a focused ion beam (FIB) method would be an alternative solution. However, the FIB method has limitations and drawbacks. The most severe problem is that a damage layer forms as a result of bombardment by highly accelerated gallium or xenon ion beams. The damage appears in the form of amorphization on the surface of the TEM foil, and this damage layer significantly degrades the quality of the TEM

This work was supported in part by NRF grants funded by the Korean government (MSIP) (Nos. 2011-0019984, 2011-0030058, and 2015R1D1A1A01059653), and in part by the Convergence Research Project (CRC-15-06-KIGAM) funded by the National Research Council of Science and Technology (NST).

(a) This is an open-access article distributed under the terms of the Creative Commons Attribution Non-Commercial License (http://creativecommons.org/licenses/by-nc/4.0) which permits unrestricted noncommercial use, distribution, and reproduction in any medium, provided the original work is properly cited.

Copyrights @ 2016 by Korean Society of Microscopy 
image. It is hard to eliminate the damage layer in practice, even though several damage reduction techniques, such as the use of a low-energy FIB, gas-assisted etching, cleaning the FIB-fabricated cross section by wet or dry etching, and cleaning by broad ion beam milling, have been suggested (Kato, 2004). Therefore, tripod polishing would be a better solution for specimen preparation of specific materials such as GaN-based, sapphire, and SiC-based structures (Chen \& Ivey, 2002). There are two typical types of tripod polishing method depending on the purpose: the flat type and wedge type. Tripod polishing can be used either as a preliminary step for the final Ar ion beam milling or as a final milling step. In addition, it can be used to prepare both cross-sectional and plan-view TEM specimens. This paper addresses flat- and wedge-type polishing methods using a tripod polisher for preparation of cross-sectional TEM specimens, especially for GaN-based and SiC-based multilayer structures, which require special techniques and care for sample preparation.

\section{TRIPOD POLISHING OF CROSS-SECTIONAL SPECIMENS}

Tripod polishing can be used either when we need to thin down the raw sample to a certain thickness that is appropriate for the final Ar ion beam milling (flat-type polishing) or when we want to directly make the specimen thin enough to be observed by TEM (wedge-type polishing). In the preparation of a cross-sectional specimen, the area of interest is usually a thin film deposited on a substrate and the interfaces between them, which are very close to the top surface of a specimen. Before the specimen is mounted on a Pyrex glass stub, therefore, it is glued to another piece of specimen or material having a hardness similar to that of the specimen using G-1 epoxy (Gatan Inc., USA) or EPO-TEK 353ND epoxy (Epoxy Technology Inc., USA). This dummy piece will protect the area of interest from being worn off preferentially during polishing. It is also important to keep the clearance (the gap between the glued materials) small when we prepare the stacked and glued specimen in order to realize secure bonding. This sandwich-like specimen is then sliced and polished using either flat- or wedge-type polishing by the tripod method.

\section{Flat-Type Polishing of Cross-Sectional Specimen}

Flat-type polishing is frequently used to prepare crosssectional specimens of Si-based devices. A flat-type specimen needs to be polished on all sides, so this method is not a good choice for $\mathrm{GaN}$, sapphire, and SiC-based structures because these materials are difficult to thin mechanically owing to their high hardness and their mechanical instability when thinned below $20 \mu \mathrm{m}$. Sapphire in particular has a tendency to crack and chip at thicknesses below $20 \mu \mathrm{m}$ (Chen \&
A

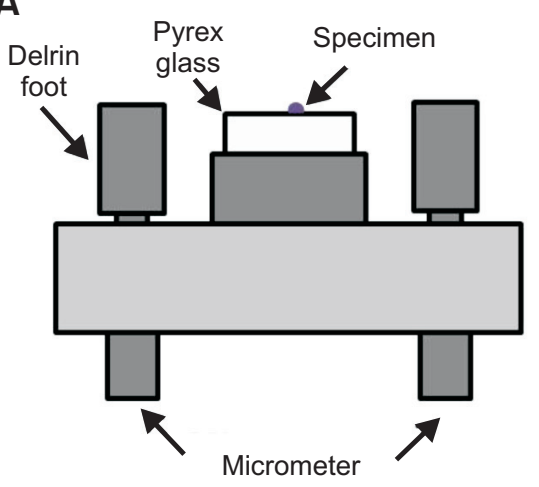

C

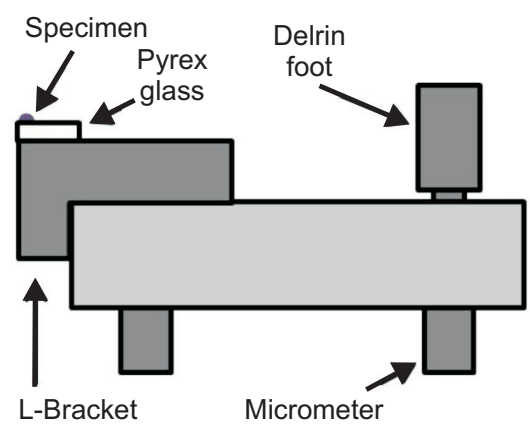

B

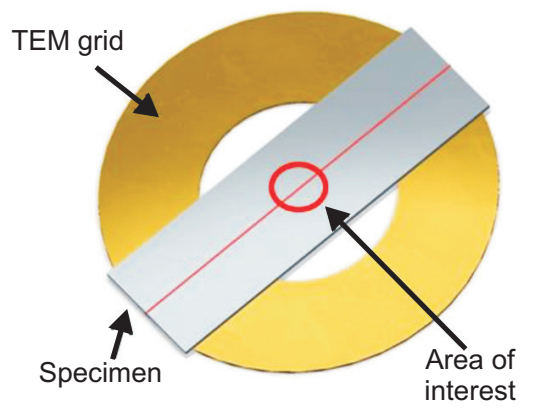

D

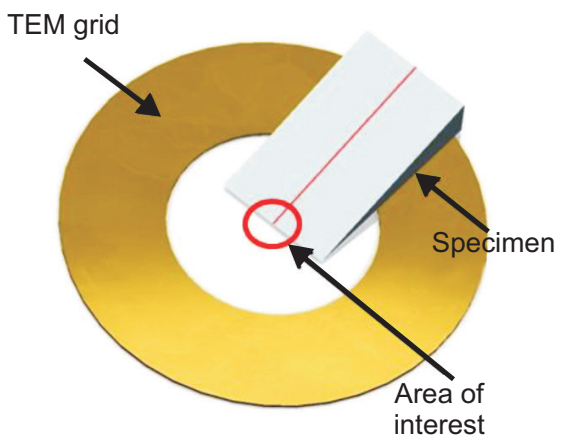

Fig. 1. Schematic of tripod polisher and specimens for flat-type (A, B) and wedgetype (C, D) polishing. TEM, transmission electron microscopy. 
Ivey, 2002). For conventional flat-type polishing, before the specimen is mounted on a Pyrex glass stub, it is stacked in a sandwich-like form. The specimen is cut into a $3 \times 1.3 \sim 1.7$ $\mathrm{mm}^{2}$ size for mounting on the Pyrex glass stub using any thermal wax, such as Mounting Wax 70 (or 52) and Crystal Bond (Electron Microscopy Sciences, USA). Fig. 1A shows a schematic diagram of the tripod polisher setup for flat-type specimens. For cross-sectional specimens, as shown in Fig. 2A$\mathrm{C}$, one side, which will be a reference plane, is first polished using diamond lapping films. A $30-\mu \mathrm{m}$ grain size diamond lapping film is used to provide a planar surface. Next, the specimen is polished using 15-, 6-, 3-, 1-, and $0.5-\mu \mathrm{m}$ grain diamond lapping films to removing material equivalent to three times the grain size from the previous step (Voyles et al., 2003). After each polishing step, the specimen is checked by an optical microscope to confirm that there are no scratches or chips. After the reference plane is polished and before the other side is polished, the specimen is lifted from the Pyrex glass stub in acetone and rinsed with ethanol. To polish the other side, the specimen demounted from the Pyrex stub needs to be mounted again such that the reference plane faces the Pyrex glass. The specimen is polished again down to 200-, 100-, 50-, and 30- $\mu \mathrm{m}$ thickness using 30-, 15-, 6-, and 3- $\mu \mathrm{m}$ diamond lapping films, respectively, as shown in Fig. 2A-C. When the specimen is $30 \mu \mathrm{m}$ thick, it is polished using a $1-\mu \mathrm{m}$ diamond lapping film until its thickness reaches $10 \mu \mathrm{m}$. Final polishing
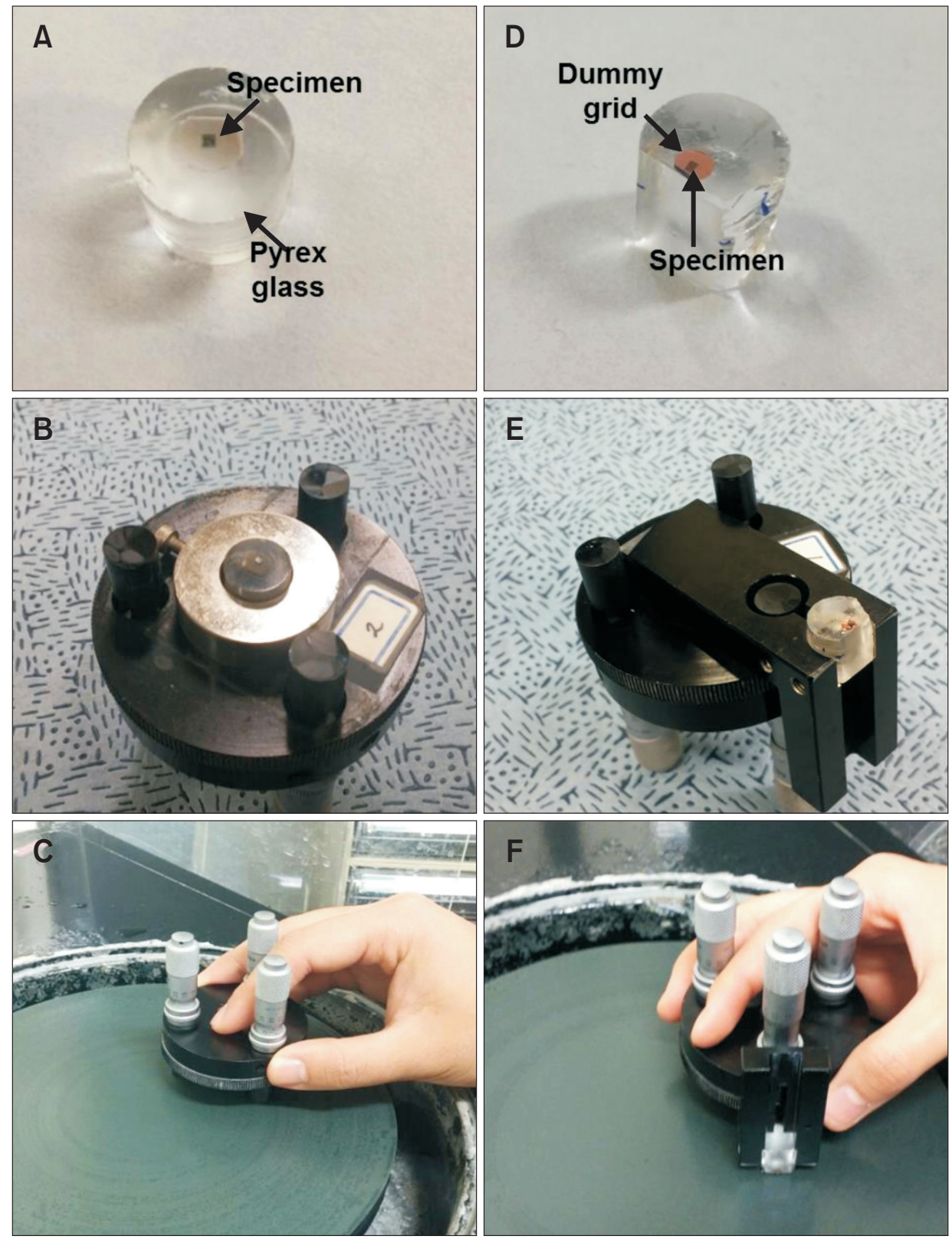

Fig. 2. Tripod polishing procedure. Reference plane preparation for flatand wedge-type polishing (A-C) and wedge-type polishing (D-F) method. (A) Mounting specimen on Pyrex glass, (B) fixing Pyrex glass on tripod, (C) polishing a reference plane, (D) remounting on edge-cut Pyrex glass, (E) fixing Pyrex glass on L-bracket, and (F) thinning the wedge specimen at a slight angle. 
is done using a $0.5-\mu \mathrm{m}$ diamond lapping film. After final polishing, the specimen is removed from the Pyrex stub and mounted on a $1.5-\mathrm{mm}$-aperture TEM grid or $2 \times 1 \mathrm{~mm}^{2}$ oval TEM grid. Fig. 1B shows a schematic of the thinned specimen. The flat-type specimen is additionally thinned to perforation using a precision ion polishing system (Gatan Inc.) or any other ion milling equipment. The milling conditions depend on the specimen material (Srot et al., 2014).

\section{Wedge-Type Polishing of Cross-Sectional Specimen}

Many steps are also involved in mechanical wedge polishing. First, a stacked sandwich-like specimen is cut into $1.5 \sim 2 \times 1$ $\mathrm{mm}^{2}$ size before it is mounted on a Pyrex glass insert. Next, as in flat-type polishing, a reference plane should be polished and prepared. After specimen mounting, the polisher is placed on the polishing machine (Fig. 2A-C). After a reference plane is prepared, the specimen is mounted on the tripod polisher using an L-bracket (Fig. 2D-F). Fig. 1C shows a schematic of the tripod polisher setup for wedge-type specimens. To polish the other side, a 3-mm TEM dummy grid is mounted on the Pyrex insert for wedge polishing using thermal wax. The edge of TEM grid should be aligned so that it just corresponds to the edge of the Pyrex glass, as shown in Fig. 3. This TEM grid acts as an indicator for estimating the amount of the polished area and the angle of the specimen. In practice, protecting the area of interest in the specimen using a dummy is important in wedge-type polishing because the specimen is frequently thinned down directly to the final thickness (a few tens of nanometers), and the dummy protects the specimen from the risk of film delamination and cracking during polishing. After the reference plane has been polished, the specimen is mounted on the TEM grid using thermal wax. An excess amount of acetone-soluble, low-melting-point thermal wax, as described above, is used to secure and protect specimens, as shown in Fig. 4 (Chen \& Ivey, 2002). There are three possible specimen mounting configurations: parallel, perpendicular, and oblique mounting, each of which has advantages and drawbacks. Fig. 3 shows a schematic of each configuration.
A

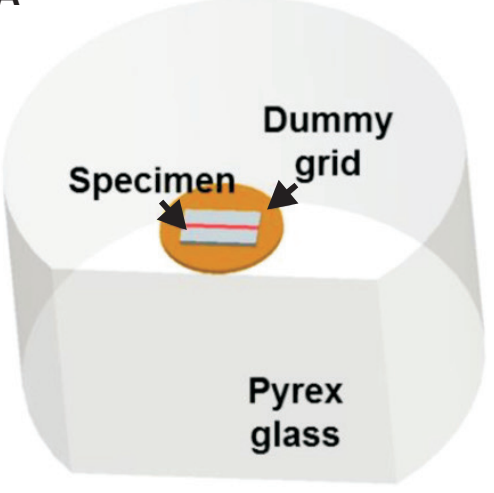

B

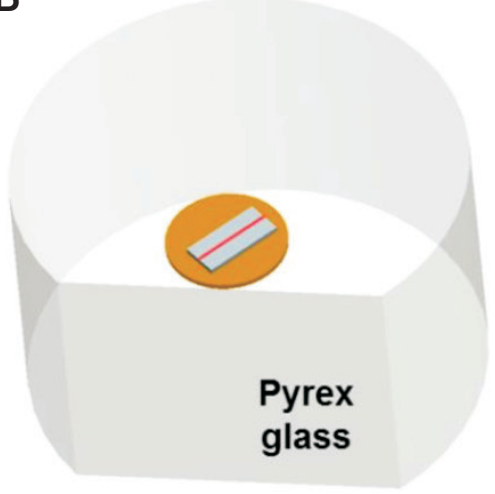

C

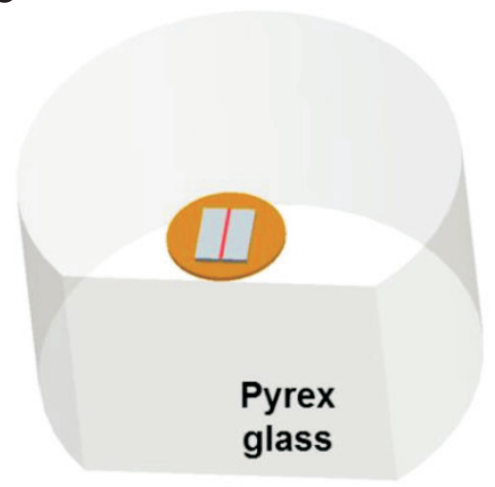

Fig. 3. Schematic of specimen mounting configurations for wedge-type polishing: (A) parallel, (B) oblique, and (C) perpendicular mounting.
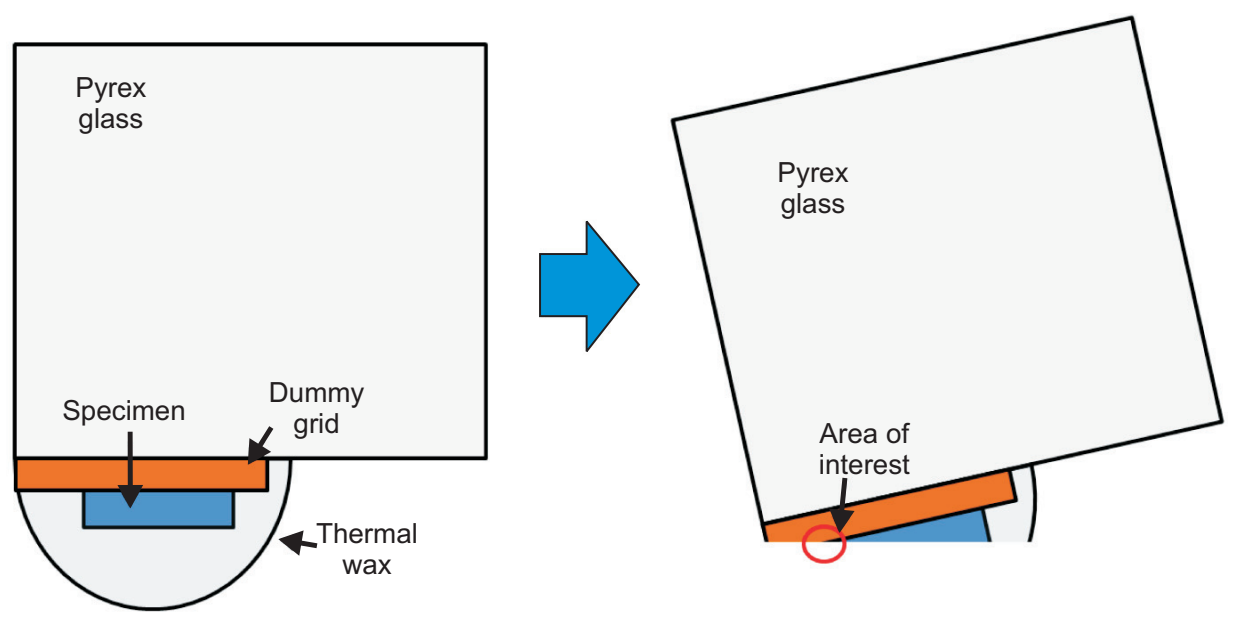

Fig. 4. Schematic of specimen mounting for wedge-type polishing. A specimen, the reference plane of which has been polished, is mounted on the dummy transmission electron microscopy grid with an excess amount of thermal wax. The dummy grid and thermal wax protect the specimen from the risk of film delamination and cracking during polishing. Then the specimen is polished at a slight angle known as the wedge angle. 
Parallel mounting can provide the largest thin area for TEM analysis, but it has the highest risk of polishing away the cross-sectional area. Perpendicular mounting can be used for multilayers/semiconductor interfaces and has a lower risk than parallel mounting, but it produces only a very small thin area of the interface. Oblique mounting is essentially a compromise between the other two techniques (Chen \& Ivey, 2002). Before wedge polishing is begun, the edge of a sample should be checked. The edge of the grid will appear doubled because of the thermal wax. After the edge of the specimen is checked, the specimen is polished at a slight angle (Fig. 4) known as the wedge angle. The specimen is polished down using a $15-\mu \mathrm{m}$ diamond lapping film until the double edge of the grid appears as a single edge. The magnitude of this angle dictates the amount of thin area that will be available in the specimen. The choice of wedge angle is a tradeoff between

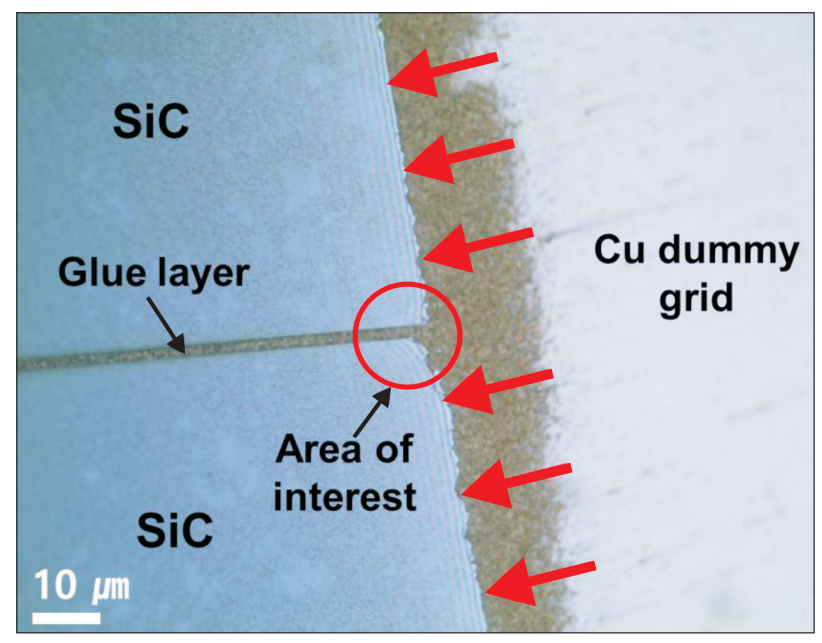

Fig. 5. Optical microscopy image of wedge-type polished $\mathrm{Ni}$-SiC sample. Thickness fringes (red arrows) appear at the edge when the specimen becomes thin enough. The thin Ni layer is not visible because of the limited resolution of optical microscopy. a large electron-transparent area (low angle) and a robust specimen (high angle) (Voyles et al., 2003). In this study, a wedge angle of approximately $2^{\circ}$ was used. By using 6- and $3-\mu \mathrm{m}$ diamond lapping films, the specimen is polished until the area of the dummy TEM grid that is scratched by the diamond lapping film approaches the edge of the specimen. Then a $1-\mu \mathrm{m}$ diamond lapping film is used to continue polishing until a thickness fringe appears at the edge of the specimen. Fig. 5 shows an example of the thickness fringes appearing at the edge of a $\mathrm{SiC}$ specimen. Final polishing is done using a $0.5-\mu \mathrm{m}$ diamond lapping film and $50-$ $\mathrm{nm}$ colloidal silica to further thin the wedge to electron transparency. Fig. 2 shows the entire process of making wedge-type specimens. After final polishing, the specimen is removed from the Pyrex and mounted on a 1.5-mm-aperture TEM grid using G-1 epoxy. A schematic of the final specimen is shown in Fig. 1D.

\section{BENEFITS AND DRAWBACKS OF EACH METHOD}

Each type of specimen preparation method has both advantages and disadvantages. The conventional method for making cross-sectional TEM specimens of semiconductors involves a dimple grinding technique in which a rotating circular dimpling tool grinds off the central part of the 3-mm disc of the TEM specimen. A TEM specimen prepared by flat-type tripod polishing has a larger area for analysis than one prepared by dimple grinding. In addition, it is easier to make a specimen using flat-type polishing. The preparation time is short compared with that of wedge-type polishing and dimple grinding. Therefore, flat-type tripod polishing can be used as an alternative to dimple grinding to achieve thinner specimens before ion milling. However, flat-type polishing has some drawbacks. In flat-type polishing, the entire specimen has to be thinned. For specimens with a substrate
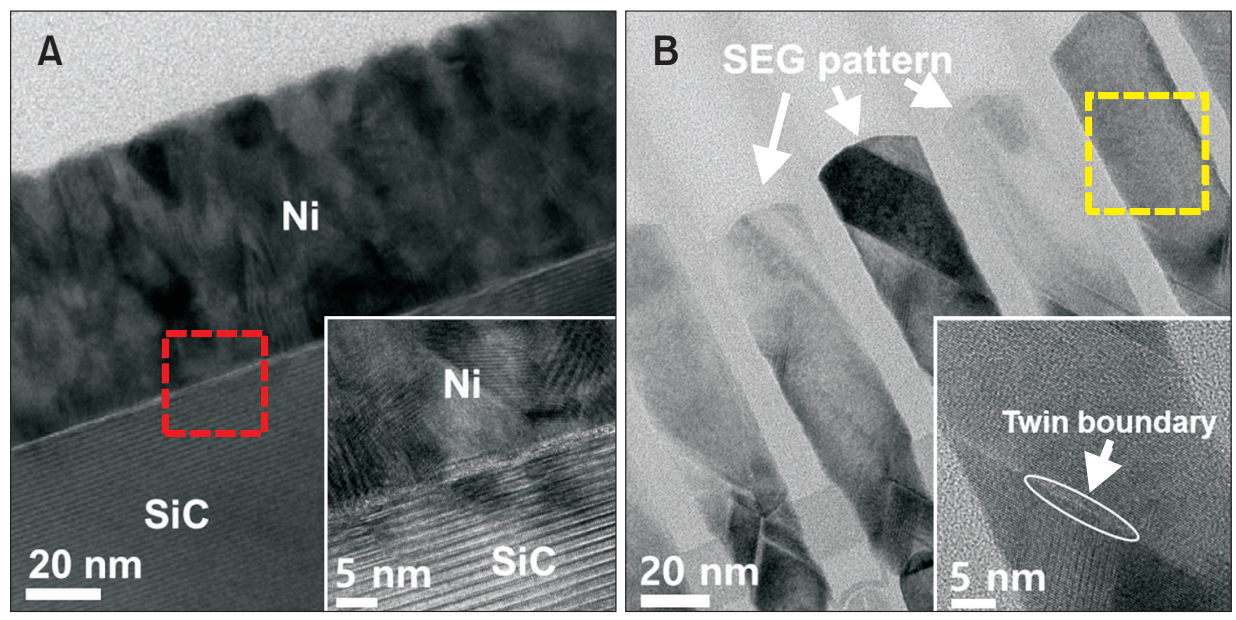

Fig. 6. Transmission electron microscopy (TEM) images of sample prepared by wedge-type polishing and low-energy ion milling. (A) Ni-SiC interface specimen. Inset is a high-resolution TEM image of $\mathrm{Ni}$-SiC interface. (B) Si-selective epitaxial growth (SEG) pattern and high-resolution TEM image (inset). 
having a different milling rate from the coated layer, or a substrate that has high hardness and mechanical instability, flat-type polishing may not be a good choice. For example, it is hard to make TEM specimens of GaN-based, sapphire, and SiC-based structures by flat-type polishing. Compared with flat-type polishing, wedge-type polishing has advantages for specimens consisting of a very limited amount of material. However, wedge-type polishing normally takes more time and is much trickier than flat-type polishing. In addition, wedgetype polishing demands extreme care because the sharp edge of the specimen, which is the area of interest, can crack and shatter easily. However, by combining tripod thinning with a final low-energy ion milling, it is possible to obtain highquality specimens for high-resolution TEM observation, as shown in Fig. 6. Fig. 6A shows a high-resolution image of a $\mathrm{Ni}-\mathrm{SiC}$ structure, of which is difficult to make a high-quality specimen using conventional methods because of a large difference in the thinning rates of $\mathrm{Ni}$ and $\mathrm{SiC}$. The uniform image contrast across the interface region allows reliable atomic-level observation. Fig. 6B shows a silicon-selective epitaxial growth (SEG) pattern. The figure shows a clear SEG pattern and high-quality high-resolution TEM image. Contamination by ion milling or a damaged layer does not appear in the images because wedge-type polishing yields a higher-quality specimen with a broader, near-electrontransparent area, which reduces the ion milling time. This figure demonstrates that combining tripod thinning with final low-energy ion milling is a superior method of specimen preparation, especially for GaN-based, sapphire, and SiCbased structures.

\section{SUMMARY}

Important progress has been made in TEM specimen preparation techniques. TEM specimen preparation is very often a major limiting factor hindering investigations. The tripod polishing method is a widely used technique for preparation of cross-sectional specimens. There are two types of specimen preparation method, the flat type and wedge type. Both types have unique advantages and disadvantages. Among the methods, a combination of wedge-type polishing and final low-energy ion milling can yield high-quality imaging of materials having high hardness and mechanical instability.

\section{CONFLICT OF INTEREST}

No potential conflict of interest relevant to this article was reported.

\section{ACKNOWLEDGMENTS}

The authors are grateful for the technical support provided by the Cooperative Center for Research Facilities (CCRF) at Sungkyunkwan University.

\section{REFERENCES}

Ayache J, Beaunier L, Boumendil J, Ehret G, and Laub D (2010) Sample Preparation Handbook for Transmission Electron Microscopy: Techniques (Springer, Berlin).

Barna A, Radnoczi G, and Pecz B (1997) Sample preparation techniques for TEM Handbook for Microscopy Vol 3 Applications, eds. Amelinckx S, Dyck van D, Landuyt van J, and Tendeloo van G, pp. 751-801, (Wiley-VCH Verlag GmbH, Weinheim).

Chen J and Ivey D G (2002) Preparation of metallized GaN/sapphire cross sections for TEM analysis using wedge polishing. Micron 33, 489-492.

Goodhew P J (1985) Thin Foil Preparation for Transmission Electron Microscopy (Elsevier, Amsterdam).

Hirsch P B, Nicholson R B, Howie A, Pashley D W, and Whelan M J (1965) Electron Microscopy of Thin Crystals (Butterworths, London).
Kato N I (2004) Reducing focused ion beam damage to transmission electron microscopy samples. J. Electron Microsc. 53, 451-458.

Klepeis S J, Benedict J P, and Anderson R M (1987) Specimen Preparation for Transmission Electron Microscopy Vol 3, eds. Bravman J C, Anderson R M, and McDonald M L, pp. 179-184, (Materials Research Society, Pittsburgh).

Srot V, Gec M, Aken van P A, Jeon J H, and Čeh M (2014) Influence of TEM specimen preparation on chemical composition of $\mathrm{Pb}$ (Mg 1/3 $\mathrm{Nb} 2 / 3) 03-\mathrm{PbTiO} 3$ single crystals. Micron 62, 37-42.

Voyles P, Grazul J, and Muller D (2003) Imaging of individual atoms inside crystals with ADF-STEM. Ultramicroscopy 96, 251-273.

Williams D B and Carter C B, (2009) Transmission Electron Microscopy: A Text Book for Materials Science (Springer, New York). 